\title{
Potential new therapeutic intervention for ischemic stroke
}

\author{
Jang-Yen $\mathrm{Wu}^{1,2,3}$, Howard Prentice ${ }^{1,2}$ \\ 'Department of Biomedical Sciences, College of Medicine, Florida Atlantic University, Boca Raton, FL 33431, USA; \\ ${ }^{2}$ Complex Systems and Brain Sciences, Florida Atlantic University, Boca Raton, FL33431, USA; \\ ${ }^{3}$ China Medical University Hospital, China Medical University, Taichung 40447, Taiwan, China
}

Stroke is one of the top leading causes of death and disability. The economic losses brought about by reduced productivity and increased social welfare spending to care for stroke survivors are in many billions of dollars. According to a 2019 update from the American Heart Association, ${ }^{[1]} 74 \%$ of the stroke cases could be attributed to behavioral risk factors, such as smoking, sedentary lifestyle, and an unhealthy diet. An estimated seven million Americans $\geq$ 20 years of age self-report having had a stroke, and the overall stroke prevalence is an approximate $2.5 \%$. Many drugs that are designed for stroke intervention and treatment are based on their antioxidative properties or as blockers of calcium channels or glutamate receptors. However, no clinically effective therapeutic intervention for stroke has been developed yet. Several neuroprotection strategies shown to be effective in animal models have failed in clinical trial. Perhaps one exception is tissue plasminogen activator (TPA), which has been widely used and is considered the gold standard treatment for ischemic stroke. However, there is a very narrow window of opportunity for TPA treatment, usually within 3-4 hours of the beginning of stroke symptoms. Furthermore, the treatment with TPA after the 3-4 hours window can increase the risk of causing dangerous bleeding in the brain causing a hemorrhagic stroke. These factors have limited its clinical application. Therapeutic intervention for stroke targeting at blocking individual molecular pathways has been proved to be inadequate for the task since the pathogenesis of stroke is multifactorial and complex involving minimally glutamate-induced excitotoxicity, oxidative stress, dysfunction of autophagy, and chronic inflammatory reactions. It is generally believed that these mechanisms are interlinked, and neuro-inflammation appears to emerge as a common factor in all these mechanisms. ${ }^{[2]}$ One better approach is to target neuroinflammatory site since inflammation requires many hours to days to fully develop and thus provides a much longer window of opportunity for treating stroke patients. ${ }^{[3,4]}$ Inflammation acts as a double-edged sword in stroke, not only augmenting secondary injury and impairing neurogenesis but also potentially promoting tissue repair. ${ }^{[5]}$ Microglia, the resident innate immune cells of the brain, are activated after ischemic stroke resulting in morphological and phenotypical changes, ${ }^{[6]}$ however, the precise mechanisms are still not clear. The sequence of events leading from cerebral ischemia to microglial responses can be summarized as follows: Step 1 is the detection by microglia of ischemia-induced cell injury and related changes in brain homeostasis such as ATP depletion, excessive glutamate level, oxidative stress, elevation of intracellular free calcium ion, dysfunction of mitochondria, and autophagy; Step 2 is the integration and transduction of signals from activated microglia into genomic or other cellular compartments resulting in pro-inflammatory effects including activation of matrix metalloproteases (MMP), for example, MMP-3 and MMP9 and production of pro-inflammatory mediators, for example, TNF $\alpha$, IL-1 $\beta$, IL-6, IL-18 or in anti-inflammatory effects including production of anti-inflammatory mediators, for example, IL-10, IL-4 , IL-13, 
and production of trophic factors, for example, BDNF, and NGF depending on the types of microglial activation. [3] One potential target for therapeutic intervention of ischemic stroke is to suppress neuro-inflammation by inhibiting microglial activation or shifting the ratio of anti-inflammatory microglia M2 to pro-inflammatory microglia M1 in favor of anti-inflammatory microglia M2. One of the molecules that appears to meet this criterion is Granulocyte Colony Stimulating Factor (GCSF). GCSF is used clinically to induce proliferation of granulocytes or antigen presenting cells for the treatment of neutropenia. We have shown that GCSF has neuroprotective and neurogenesis functions in Parkinson's disease (PD) ${ }^{[7]}$ and cerebral ischemia stroke animal models. ${ }^{[8-10]}$ Furthermore, we have demonstrated that the neuroprotective function of GCSF is in part mediated through its anti-inflammatory response since rapamycin, an inhibitor of $\mathrm{mTOR}$, abolished both the neuroprotective effects and its anti-inflammatory responses such as increased level of anti-inflammatory cytokine, IL-10. ${ }^{[10]}$ In addition to GCSF protein, we have recently shown that GCSF gene therapy, namely AAVGCSF gene vectors delivered via eyedrop is as effective as the GCSF protein therapy for treatment of ischemic stroke. ${ }^{[1]}$ The new target for therapeutic intervention for stroke as discussed above using GCSF protein or GCSF gene therapy targeting the immune system is attractive and appealing as summarized below: (1) TPA treatment for ischemic stroke is considered to be the gold standard treatment for ischemic stroke, it has a serious limitation due to a very narrow time window of opportunity for TPA treatment, usually within 3-6 hours of the beginning of stroke symptoms and its use may increase the risk of hemorrhage. These factors have limited its clinical application. By contrast, brain inflammation develops over a much slower time course and requires many hours to days to fully develop. Thus, the therapeutics such as GCSF or GCSF gene therapy, which target the immune system via microglia and mTOR, will provide not only neuronal protection but also neurogenesis over the course of disease progression and recovery. (2) Unlike any of the pharmacological interventions currently available, GCSF or GCSF gene therapy provides a new and innovative twopronged approach to preserve and restore the function of the brain by first protecting the brain against strokeinduced injury through its neuroprotective function, and secondly, by stimulating neurogenesis to replenish new brain cells. ${ }^{[9,11]}$ (3) Moreover, GCSF gene therapy could provide a sustainable and continuous supply of GCSF from the AAV-GCSF vectors. We have demonstrated the efficacy of GCSF gene therapy in the BCAO stroke model. ${ }^{[11,12]}$ The advantage of gene therapy versus protein therapy is that the gene therapy can provide a much longer life of the expression of the protein whereas the protein therapy usually is limited by the relative short life of the protein (for GCSF the half-life is less than 4 hours). In addition, the level of GCSF expression in the brain could be well regulated since the vector to be used in the gene therapy includes the Hypoxia Response Element (HRE) within the promoter domain, which is regulated by the hypoxic condition, while the severity of stroke is known to be related to the hypoxic condition. (4) The method of viral administration via eyedrop is simple, non- invasive, and clinically relevant. We have verified the presence and expression of human GCSF in the brain in stroke mouse model after the delivery of AAV-hGCSF via eyedrop by non-invasive MRI as well as by RT-PCR. ${ }^{[12]}$

\section{Source of Funding}

This research was supported by grant 09KW-11 and 6JK08 from the Department of Health, James and Esther King Biomedical Research Program, State of Florida.

\section{Conflict of Interest}

The authors declare that there is no competing interest.

\section{REFERENCES}

1. Benjamin EJ, Muntner P, Alonso A, Bittencourt MS, Callaway CW, Carson AP, et al. Heart Disease and Stroke Statistics-2019 Update: A Report From the American Heart Association. Circulation 2019; 139: e56-e528.

2. Anrather J, Iadecola, C. Inflammation and stroke: An Overview. Neurotherapeutics 2016; 13: 661-70.

3. Yenari, MA, Kauppinen, TM, Swanson RA. Microglial activation in stroke: Therapeutic targets. Neurotherapeutics 2010; 7: 378-91.

4. Wang J, Xing H, Wan, L, Jiang X, Wang C, Wu Y. Treatment targets for M2 microglia polarization in ischemic stroke. Biomed Pharmacother 2018; 105: 518-25.

5. Jayaraj RL, Azimullah S, Beiram R, Jalal FY, Rosenberg GA. Neuroinflammation: friend and foe for ischemic stroke. J Neuroinflammation 2019; 16: 142.

6. Guruswamy R, ElAli A. Complex roles of microglial cells in ischemic stroke pathobiology: new insights and future directions. International journal of molecular sciences. Int J Mol Sci 2017; 18: 496.

7. McCollum M, Ma Z, Cohen E, Leon R, Tao R, Wu JY, et al. PostMPTP treatment with granulocyte colony-stimulating factor improves nigrostriatal function in the mouse model of Parkinson's disease. Mol Neurobiol 2020; 41: 410-9.

8. Menzie-Suderam J, Gharibani P, Modi J, Ma Z, Tao R, Prentice H, et al. Granulocyte-colony stimulating factor protects against endoplasmic reticulum stress in an experimental model of stroke. Brain Res 2018; 1682: 1-13.

9. Modi J, Menzie-Suderam J, Trujillo P, Medley K, Marshall M, Tao R, et al. Mode of Action of Granulocyte-colony Stimulating Factor (G-CSF) as a Novel Therapy for Stroke in a Mouse Model. J Biomed Sci 2020; 27: 19-37.

10. Dumbuya JS, Chen L, Shu SY, Ma L, Luo W, Li F, et al. G-CSF attenuates neuroinflammation and neuronal apoptosis via $\mathrm{mTOR} / \mathrm{p} 70 \mathrm{~S} 6 \mathrm{~K}$ signaling pathway in neonatal hypoxia-Ischemia rat model. Brain Res 2020; 1739: 146817. 
Wu and Prentice: Novel therapeutic intervention for ischemic stroke

11. Menzie-Suderam J, Modi J, Xu H, Bent A, Trujillo P, Medley K, et al. Granulocyte-colony Stimulating Factor Gene Therapy as a Novel Therapeutics for Stroke in a Mouse Model. J Biomed Sci 2020; 27: 99.

12. Ren J, Chen YI, Liu CH, Chen PC, Prentice H, Wu JY, et al. Noninvasive tracking of gene transcript and neuroprotection after gene therapy. Gene Ther 2015; 23: 1-9.

How to cite this article: Wu JY, Prentice $\mathrm{H}$. Potential new therapeutic

intervention for ischemic stroke. J Transl Intern Med 2021; 9: 1-3. 